\title{
Potential Role of Biochemical Components of Schisandra chinensis for Prevention and Treatment of Obesity, Type 2 Diabetes Mellitus, Cancer and Prevention of Aging - A Systematic Review
}

\author{
Kulvinder Kochar Kaur ${ }^{1 *}$, Gautam Allahbadia ${ }^{2}$ and Mandeep Singh ${ }^{3}$ \\ ${ }^{1}$ Scientific Director, Dr Kulvinder Kaur Centre for Human Reproduction, Jalandhar, Punjab, India \\ ${ }^{2}$ Scientific Director, Rotunda-A Centre for Human Reproduction, Mumbai, India \\ ${ }^{3}$ Consultant Neurologist, Swami Satyanand Hospital, Jalandhar, Punjab, India \\ *Corresponding Author: Kulvinder Kochar Kaur, Scientific Director, Dr Kulvinder Kaur Centre for Human Reproduction, Jalandhar, \\ Punjab, India.
}

Received: June 24, 2019; Published: July 31, 2019

DOI: $10.31080 /$ ASNH.2019.03.0388

\begin{abstract}
The growing incident of diabetes mellitus (DM) and obesity globally has made us look for novel treatment protocols utilizing natural products. Earlier we have reviewed the use of natural plant derivatives like flavonoids, anthrocyanins, terpenoids, essential oils, or products from aquatic species to determine the biochemicals that can be utilized for treatment of obesity and T2DM and as a sequel cancer resulting secondary to obesity. Since Schisandra chinensis (SC) Baill is a plant whose components have been shown to have all these properties and in addition antimicrobial properties and antiaging ones we decided to carry on a systematic review on components of S. Chinensis. Thus we carried out a pubmed search for MeSH terms like Schisandra chinensis, its components schisandrin A, B, Gomisin A, B and their biochemical actions with respect to antidiabetic action, effect on glucose and lipid metabolism, obesity, adipogenesis, different glucose transporters till 2019.We found a total of 150 articles out of which we selected 83 articles for this review. No meta-analysis was carried out. We found that the components of SC(SCE) not only had a protective effect on obesity by preventing lipid accumulation and differentiation of adipocytes, but antidiabetic properties by improving hyperglycemia through different mechanisms including altering SGLT2 and GLUT 2 transporters modulating AMPK activity, acting as PT1B inhibitors. Further they had antimicrobial effects and exerted a protective effect against skin photoaging, osteoarthritis, sarcopenia senescence along with mitochondrial dysfunction and improved physical endurance. Along with cognitive/behavioral functions that can be associated with its general antiaging effects. Normally in food technology SCE is currently used for increasing flavor and taste. Along with nutritional value of food. Thus one needs to isolate the constituents responsible for its benefits so that they can be utilized for human health.
\end{abstract}

Keywords: SC; T2DM; Obesity; Cancer; Antimicrobial Actions; Antiaging Effects

\section{Introduction}

Natural plants have been utilized for nutrition, food production and medicine for several years. Many contain an array of compounds having antimicrobial, antioxidative, antiproliferative and anticancer activity [1]. These natural plant extracts have the potential to induce pro health effects resulting in an extension of life expectancy and improvement of its quality. Plant extracts and plant derived compounds can improve the properties of functional food with their well-documented pro-health effects [1].

Schisandra chinensis (Turcz). Baill. (SCE)is a plant whose fruit have longstanding use in traditional Chinese medicine. Their use has been done in gastrointestinal tract (GIT), respiratory failure, cardiovascular disease (CVD), body fatigue and weakness, exces- 
Potential Role of Biochemical Components of Schisandra chinensis for Prevention and Treatment of Obesity, Type 2 Diabetes Mellitus, Cancer and Prevention of Aging - A Systematic Review

sive sweating and insomnia [2]. Further they decrease hunger, delay aging, increase vitality and improve mental health [3]. They show neuro and hepatoprotective, anti-inflammatory, antioxidative, detoxification, immunostimulant, antiviral, and anti cancer activities along with CVS and skin protective properties. The aim of this review is to describe the SCE effects on CNS, sympathetic, CVS, endocrine, respiratory systems along with its adaptogenic, hepatoprotective, immunostimulant, antioxidant, ergogenic and anti-stress activities [2-4].

Active compounds in SCE

Schisandra chinensis has many active compounds that include lignans, triterpenes, phenolic acids, flavonoids, essential oils and polysaccharides. Lignans are the ones responsible for their prohealth properties. They are mainly present in SCE fruits, but can also be found in leaves, shoots and seeds. They were extracted from the biomass of in vitro cultures [5]. The most widely represented group of SCE lignans are dibenzocyclooctadiene lignans, which due to structural similarity to and occurrence in plants of the Schisandra genus are often called "Schisandra lignans". Within dibenzo cyclooctadiene lignans that occur in larger quantities in fruits of Schisandra chinensis, schisandrin(syn, Schisandrol A, wuweizisu A), Schisanderin B(gomisin, wuweizisu B, $\gamma$ - schisandrin) Schisantherin A (syn gomisin C, schisandrin A, schisadrer B), and gomisin B(syn Schisanhenol (syn gomisin K3), deoxy schisandrin (syn schisandrin A) and gomisin A (syn Schisandrol (B) [3]. The World health organization (WHO) monograph said that about 30 Schisandra lignans were found, but to ensure the prohealth activity of fruits, their content needs to be lower than $0.4 \%$. At present many more lignans have been found. E. g. schineolignins A-C, belonging to the butane type lignans were isolated by Yang., et al. [6] from rattan stems of SCE. Further chemical composition and resulting biological activity of plant varies with humidity, light, latitude, season maturity, harvest time, geographical location and other factors [7].

Another important group detected from SCE is triterpenoids. SCE contains lanostane and cycloartane-type triterpenoids and non triterpenoids, that in the scientific literature are often called Schisandra non triterpenoids or schinor triterpenoids [8]. Eg of a lanostane type triterpenoids is kadsuric acid, described by Yang., et al. [9]. E.g's of cycloartane- type triterpenoids are schisanlactone D and wuweizilactone acid [9]. isolated from different parts of plant- fruits (schindilactone A, wuweizidilactone I, leaves (schindilactone IK, wuweizidilactone IP, scisanartanin $\mathrm{N}$ ) rattan stems (schindilactone IM, wuweizidilactone S) and roots (Shinchinelactone D) [9].

Flavonoids and phenolic acids, that are polyphenols, show antioxidant properties. They are secondary plant metabolites that occur in every part of plant (like fruit, flowers, seeds, leaves, roots or even identified parts). Among phenolic acids, Mocan., et al. found chlorogenic acid in the fruits of SCE, while in the leaves two other derivatives of hydroxy cinnamic acid (p-cumaric and feruluc) were found. Significantly more compounds from this group were found by Szopa., et al. [10]. Nowak., et al. [7] found chlorogenic acid and five hydroxyl benzoic acid derivatives: gallic, p-hydroxy benzoic, protocatechuic, syringic, and vanilic acids in the leaves and fruits. Flavonoids present in SCE fruits were isoquercetin, quwercetin, and its derivatives -quercetin-3-galactoside (hyperoside) and quercetin 3 rutinoside (rutin). SCE Leaves also contain quercetin 3-ramnoside(quercitrin) myricetin and kaempfenol [10]. Fruits of SCE also comprise the cyanidin derivatives: cyanidin xylosylrutinoside, cyanidin glocosylrutinoside and cyanidin- xylosylglucoside and cyaniding-rutinoside, belonging to the anthocyanins [11].

SCE fruits also contain essential oils. Detailed amounts of terpenes reviewed in Nowak., et al [7].

Also, homogenous polysaccharides present in SCE fruits contain mainly glucose, galactose, mannose and rhamnose in different molar proportions, with mass varying from 18-127kDa [12]. Polysaccharides also occur in combination with uronic acid and proteins [13].

SCE fruits as shown by Sowndharajan., et al. that $100 \mathrm{~g}$ of dried fruits contain $\mathrm{Fe}, \mathrm{Mn}, \mathrm{Cu}, \mathrm{K}$, and $\mathrm{Mg}$ in amounts which cover 96\%, $320 \%, 48 \%, 54 \%$ and $33 \%$ of the Recommended Daily Intake (RDI) of these ingredients respectively [14]. As per the European Union Legal regulations, a food can be treated as a source of particular substance if it contains $>15 \%$ of the RDI of the substance in $100 \mathrm{~g}$ of the product.

\section{Health effects}

\section{Antimicrobial activity}

SCE berry extract has shown antibacterial actions against various gram-positive and gram -negative bacteria. Oils from SCE seeds exhibited good antibacterial actions against Enchiridia Coli. 
Potential Role of Biochemical Components of Schisandra chinensis for Prevention and Treatment of Obesity, Type 2 Diabetes Mellitus, Cancer and Prevention of Aging - A Systematic Review

Bacillus cereus, Enterobacter aerogenes, Serratia marcescens and Micrococcus luteus as tested by the disc diffusion method. Simultaneous distillation extracted >amounts of terpenes, $\beta$-pinenes, borneol, and $\alpha$ pinenes, along with limonene than other procedures that included Soxhlet and microwave assisted extraction as shown by Teng and Lee [15]. These compounds might show a strong antibacterial actions in view of penetration through the outer membrane of bacterial cells and its severe damage. Six dibenzo cyclooctadiene lignans presented antibacterial activity against pathogenic Chlamydia pneumoniae and Chlamydia trachomatis upon their infection in human epithelial cells [16]. Presence and substitution pattern of methylenedioxy, methoxy and hydroxyl groups of lignans had a profound effect on anti-Chlamydial activity [16]. Activity of SCE fruit ethanolic and water extracts against typical food borne pathogens and food -spoiling organisms. Both extract s showed strong antibacterial actions against Staphylococcus aureus, Listeria monocytogenes, Bacillus subtilis, B. cereus, Salmonella enteric subsp enteric a serovar Typhimurium, Pseudomonas aeruginosa, Enterobacter aerogenese and E. Coli [17]. It was suggested that the main constituents responsible for this activity were organic acids (like citric acid and malic acids) as checked by ion chromatography. Minimal inhibitory concentration (MIC) of $S$. chinensis fruit and leaf extracts was tested by Mocan., et al. for the gram-positive Staphylococcus aureus, Bacillus subtilis and Listeria monocytogenes and gram -negative bacteria E. coli and $S$. Typhimurium, that ranged from $10 \mu \mathrm{g} / \mathrm{mlto}>100 \mu \mathrm{g} / \mathrm{ml}$ [15]. These results also indicated that gram positive bacteria are more sensitive to SCE extracts than gram negative bacteria. Choi., et al. found same findings regarding methanol fractions of SCE against several gram -negative (E. coli and S. typhimurium, Cronobacter sakazaki) and gram positive Staphylococcus aureus, Bacillus subtilis and Listeria monocytogenes strains [16]. This difference might result from the difference in cellwall morphology of these microorganisms [16]. Reports on the stimulation of microbial growth by compounds from SCE are less [16]. In a conference report S, chinenze rhizome extract was reported to promote the growth of Lactobacillus delbrueckii ssp bulgaris, while inhibiting activity of Bacillus licheniformis, $B$, subtitis and the pathogenic E. coli [17]. The mechanism of inhibition includes changing the permeability of the outer membrane of bacteria, leading to their destruction [17].

\section{Anti cancerous activity}

The anticancer activity of polyphenols from plant extracts in cancer lines include different mechanisms; inhibition of tumor proliferation, induction of cell death(apoptotic, autophagic)inhibition of tumor migration and invasion, cell cycle arrest, prooxidant activity by stimulation of ROS, production in cancer cell lines, as well as reducing oxidative stress in normal cells and inhibition of carcinogenic activity [18]. (Figure 1) Cytotoxic (antiproliferative) activity of SCE main constituents such as gomisin N, was shown against many cancer cell lines [2,19]. On overdosing, SCE is toxic -the minimal toxic dose when given orally to mice is $3.6 \mathrm{~g} / \mathrm{kg}$ [3]. Normal cell lines in reaction to plant derived chemical compounds (like phytochemicals and essential oils) can behave differently to cancer cells [20]. Limited data exist on the cytotoxicity and genotoxicity of Schisandra extracts or its constituents on normal cell lines, compared to their cancer counterparts. Anticancer activity of gomisin, was shown by Kee., et al. via inhibition of proliferation of several colorectal cancers cell lines while that compound did not change the proliferation of normal colon cells [21]. Some studies show that the main constituents of SCE to induce cell cycle arrest and apoptosis in cancer cells by ROS mediated/mitochondria dependent pathway [22]. Schisandrin B has been shown to protect against oxidative damage in liver, heart an brain tissues in rodents. Cell migration and invasion are critically involved in cancer metastases, the main cause of death in cancer patients. In in vivo research Schisandrin B attenuated cancer invasion and metastases in BALB/c mice (an albino, laboratory bred strain of the house mouse useful for research into both cancer and immunology [23]. In in vitro studies Schisandrin B inhibited the invasion and migration of the human alveolar basal epithelial adenocarcinoma cell lines (A549) by downregulating the expression of hypoxia inducible factor (HIF-1), Vascular endothelial growth factor (VEGF), and matrix metalloproteinases (MMP2 and MMP9) [23]. Then gomisin A, decreased invasion and migration of colorectal cancer cell lines, as well as the metastases in BALB/c mouse lung [22]. The research relevant to mechanisms of anticancer actions of cytotoxicity and apoptosis of SCE in cancer cells is advanced, while data on normal cells is insufficient. 


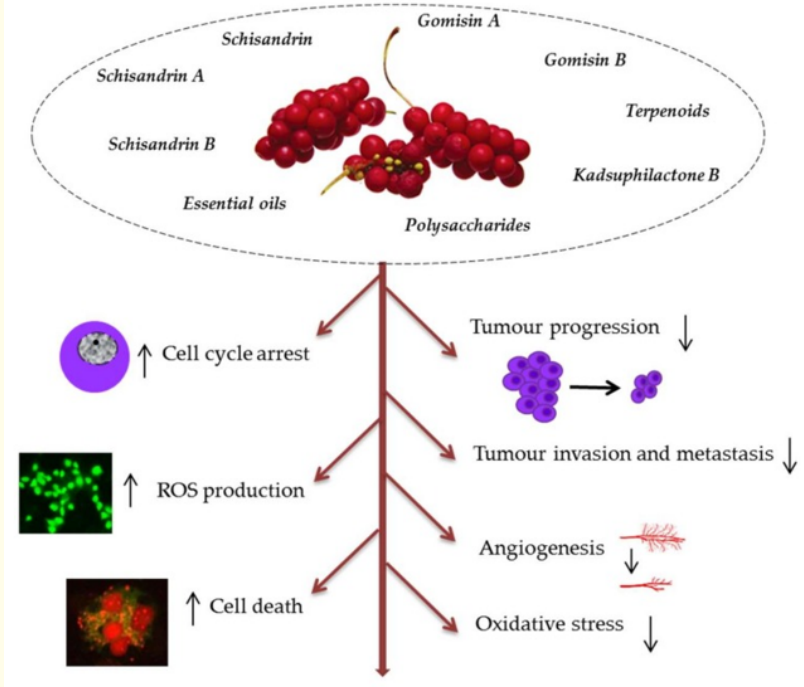

Anti-cancer effects

Figure 1

Courtesy ref no-7. Mechanisms of anti-cancer activity of bioactive phytochemicals in Schisandra chinensis (SCE). They may inhibit tumour progression through cell cycle arrest at G0/G1 and $\mathrm{G} 2 / \mathrm{M}$, suppression of proliferation, invasion, metastasis, and angiogenesis. SCE antioxidative action includes induction of the antioxidant enzymes and direct scavenging of reactive oxygen species (ROS) to prevent cancer induction and progression. Their pro-oxidant effects lead to increased ROS production in cancer cells and cell death (apoptotic and autophagic).

\section{Anti obesity and Anti DM effects}

In view of its antioxidant, hepatoprotective, and anticancer activities, SCE fruit has been used as a traditional medicine for treatment of various CVS or GIT ailment in South Eastern Asia and Russia [24]. Its application as a preventive agent against diet related chronic diseases such as type2 diabetes (T2DM), obesity or nonalcoholic fatty acid disease (NAFLD) has increased recently.

\section{Carbohydrate metabolism modulation by SCE}

Chronic elevation of blood glucose or postprandial hyperglycemia constitutes one of major features of T2DM. Mostly following a meal intake, glucose is released from carbohydrates in the digestive tract. This reaction is catalyzed mainly by $\alpha$-amylase, that hydro- lyzes starch to maltose and maltotriose, as well as $\alpha$-glucosidase, that catalyses glucose release from disaccharides and oligosaccharides. Hence the inhibition of these activities is used as the 1st therapeutic target to control blood glucose level. Jo., et al. checked the inhibitory potential of 2 SCE water polyphenolic extracts, fruit pulp, or skin and seeds and showed that the former was a potent inhibitor of porcine pancreatic $\alpha$-amylase and rat intestinal $\alpha$-glucosidase (figure 2) [25]. These hypoglycaemic properties were further tested, in in vivo studies, when a SCE preparation reduced blood glucose levels in rats after sucrose solution oral administration, with an efficiency greater than that seen with acarbose that is a known $\alpha$-glucosidase inhibitor.

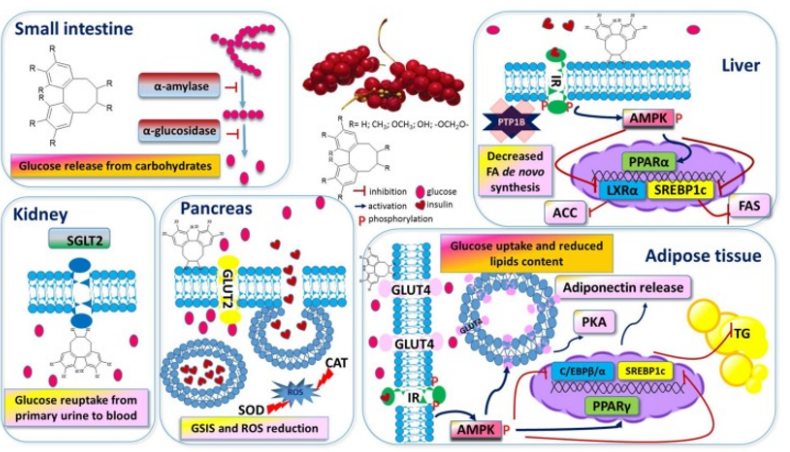

Figure 2

Courtesy ref no-7. Proposed molecular mechanisms of antidiabetic and anti-obesity actions of Schisandra chinensis in different organs and adipose tissue. See main text for more details. ACC-acetyl-CoA carboxylase; C/EBP $\beta / \alpha$-CCAAT/enhancerbinding protein alpha; CAT_catalase; FAS—fatty acid synthase; GLUT2, 4-glucose transporter 2, 4; GSIS—glucose stimulated insulin secretion; HSL-hormone sensitive lipase; IR-insulin receptor; LXR $\alpha$-liver X receptor $\alpha$; PKA-protein kinase A; PTP1B-protein tyrosine phosphatase 1B; ROS-reactive oxygen species; SOD—superoxide dismutase; SREBP-1c-sterol regulatory element binding protein $1 \mathrm{c}$; and $\mathrm{TG}$-triglyceride.

Blood glucose level is strongly influenced by transport and absorption in the small intestine and transportion in the kidneys, as well as uptake in other peripheral tissues. During this process 2 types of membrane-integrated transporters, called glucose trans- 
porters (GLUTs) or sodium glucose cotransporters (SGLT's) are involved [26]. SGLT1 is involved in intestinal glucose absorption, while SGLT2and SGLT1 takes part in renal reabsorption of filtered glucose from the primary urine. In diabetic patients secondary to raised SGLT2 expression, there is increased renal glucose reabsorption=> hyperglycemia. Hence SGLT2 transporters are considered for potential DM treatment targets. Fractions obtained from SCE fruit ethanol extract enriched with lignans -deoxy schisandrin, schisandrin B and schisandrin-selectively inhibited glucose reuptake by SGLT2, that finally eliminated glucose excess via urine $=>$ reduction in blood glucose level $[27,28]$. Of the known GLUTs responsible for the uptake of different monosaccharides, the GLUT4isoform is the principal protein involved in glucose transport into insulin sensitive tissues [28]. In T2DM patients, the cellular expression of GLUT1 is decreased, indicating a lower capability to process glucose in vivo. GLUT4 is mainly expressed in muscle cells and adipocytes, but recently it has been found in other types of cells [27]. A low molecular weight polysaccharide fraction extracted from SCE upregulates the expression of GLUT4 in fibroblasts of hepaticorigin (Buffalo rat liver) and improves glucose uptake has been shown [27]. But cellular glucose uptake by GLUT4 transporter needs translocation from intracellular space to the plasma membrane and is regulated by 5 'adenosine monophosphate activated protein kinase (AMPK) [29]. AMPK is one of the most important regulators of lipid and glucose metabolism and is regarded as a potential target in the prevention and treatment of DM (figure 2). AMPK is activated via its phosphorylation under metabolic stress, when ATP consumption and of AMP:ATP ratio increase. That the hyperglycaemia activity of SCE polysaccharides was associated with the increase of pAMPK protein level, as well as mRNA of IRS-1, GLUT4, AMPK $\alpha$, and PPAR $\gamma$ levels [27]. Conversely, dephosphorylation of the insulin receptor (IR) and insulin receptor substrate 1 (IRS1) by another enzyme, protein tyrosine phosphatase 1B(PTP1B), interrupts the insulin signaling pathway. Hence PTP1B inhibitors increase insulin sensitivity and glucose tolerance, and a petroleum ether extract of SCE is recognized as a PTP1B phsphatase inhibitor [30].

Pancreatic beta cells secreting insulin play a critical role in glucose homeostasis. In view of intrinsic low level and the minimum activity of the antioxidant enzyme catalase (CAT), Glutathione peroxidase, and super oxide dismutase (SOD), these cells are very sensitive to oxidative stress induced by increased levels of glucose and free fatty acids [31] (Figure 2). Hence the high antioxidant activity and radical-scavenging capacity of SCE extracts play an important role in the protection of $\beta$ cells against their dysfunction or death It was observed in diabetic rats, that SCE oil supplementation improved pancreatic- $\beta$ cells function by upregulation of SOD and CAT, along with the expression of the anti-apoptotic Bcl2 gene [46]. Further due to the hyperglycemia and oxidative stress, insulin release from granules by $\beta$ cells is impaired along with loss of pancreatic mass were seen [32]. Insulin acts as an agonist for IR's and in turn activates further glucose uptake by other peripheral tissue, including adipocytes, via GLUT4. A water extract of SCE showed insulinotropic action to improve glucose-stimulated insulin secretion by mouse Min6 cells [32]. But on further analyses on diabetic rats, subjected to overnight fasting, suggested that a SCE preparation improved glucose homeostasis by the promotion of insulin sensitivity but not secretion capacity [32]. In accordance with this more recent studies show SCE protective activity against the production of advanced glycation end-products (AGEs), that are harmful to endothelial cells [48]. AGE's are produced through nonenzymatic reactions during hyperglycemia and promote ROS production, that attenuates the expression and activity of endothelial nitric oxide, and finally in the formation of human umbilical vein endothelial (HUVEC) cell incubation with SCE increased the expression and activity of eNOS through downregulation of Rho A/Rho kinase activity; therefore SCE was able to induce hyperglycaemia induced microvascular complications.

\section{Lipid Metabolism modulation}

During obesity accumulation of excess fat in adipose tissue (AT), and its development occurs along with the differentiation of preadipocytes into adipocytes. The process is called adipogenesis, that can be seen in vitro by the increased ability of adipocytes to accumulate triacyl glycerol in cytosolic lipid droplets. Schisandrin B decreased lipid content, along with increased acid oxidation and lipolysis, with the activation of protein kinase A (PKA) and hormone sensitive lipase (HSL) in 3T3L1 adipocytes [33] (figure 2). A similar influence of Schisandrin B on subcutaneous mouse adipocytes in an exvivo model was shown by the same experiment. In an vivo experiment Schisandrin B decreased subcutaneous adipocytes size, subcutaneous adipocytes tissue mass and the body weight in mice.

Major adipogenesis regulator is peroxisome proliferator activated receptor gamma (PPAR- $\gamma$ ) (figure 2). Insulin sensitizers are 
its agonists, able to enhance triacyl glycerol storage in AT by increasing insulin-stimulated glucose uptake. Sterol regulatory element binding protein 1c (SREBP1c) is another transcriptional factor involved in lipid metabolism. Activation of PPAR- $\gamma$ and SREBP1c regulates the expression of other genes which encode proteins responsible for fat cell development, considered as a positive effect that will stimulate $\beta$-oxidation, insulin sensitivity, adiponectin secretion, glucose uptake and catabolism. SCE fruit extract, enriched with Schisandrin, gomisin $\mathrm{A}$, and angeloyl gomisin $\mathrm{H}$, acted as a PPAR- $\gamma$ agonist along with improving insulin resistance in the liver. But further studies demonstrated that SCE extract and its lignans prevented lipid accumulation and impaired the differentiation of 3T3L1 preadipocytes into adipocytes, via downregulation of the key adipocytes differentiation regulatory genes PPAR- $\gamma$ and C/ EBP $\beta / \alpha$. SCE anti-obesity properties observed in vitro were comparable to results obtained in high fat diet (HFD)-induced obese rats. The liver plays a major role in maintaining normal blood glucose levels by regulating de novo glucose production and triglycerides (TG) accumulation (by impairing insulin mediated inhibition of gluconeogenesis and regulating insulin mediated TG-metabolism, respectively), which contributes to hyperglycemia and dyslipidaemia. Thus control of hepatic IR is an attractive therapeutic target for treating T2DM and hepatic steatosis. Studies done on hamsters treated with HFD, showed that SCE ethanol extract reduced food and energy intake body and fat weights (as well as the serum TG and LDL levels) IR, inflammation and liver steatosis [24]. The amelioration of liver lipid accumulation was due to the increase of PPAR- $\alpha$ mRNA level and reduced transcription of lipogenic SREBP1c which promotes limited synthesis in the liver and is involved in triacylglycerol synthesis via fatty acid synthetase (FAS) and acetyl CoA Carboxylase (ACC) (figure 2). At the protein level, the extract considerably increased the AMPK phosphorylation(pAMPK), that acted cellular lipid oxidation allowing ATP generation with the simultaneous exclusion of energy -consuming processes, like TG and protein synthesis. Moreover, a SCE lignan extract inhibited FAS enzymatic activity.

Chronic increased lipid accumulation in the liver =>the development of nonalcoholic fatty liver disease (NAFLD). SCE water extract lowered hepatic triglyceride, total cholesterol, glucose content, and mitigated ALT (Alanine amino transferase) released by liver release by a liver injured by fenfibrate (a PPAR- $\alpha$ agonist) in normal and hypercholesteraemic mice [33]. SCE ethanol extract at- tenuated palmitic acid and oleic acid induced lipid accumulation in human hepatoma HepG2 cells shown in recent studies. SCE reduced the expression of ER stress markers and inflammatory genes encoding IL-6, TNF- $\alpha$, and MCP-1 proteins as shown by Zhu., et al. while Chung., et al. showed that SCE berry ethanol extract attenuated lipid accumulation by the inhibition of histone acetyl transferase (HAT) via inhibition of total lysine acetylation [33,34]. Reduced levels of Ppar $\gamma$, Srebp1c and Fas gene expression was found by these authors in mice supplemented with SCE [34]. The hepatoprotective activity of SCE was also shown by a polysaccharide fraction, which downregulated mRNA and protein expression of SREBP1c, FAS, ACC and liver X receptor $\alpha(L X R \alpha)$ in mice with induced NAFLD. Additionally gomisin $\mathrm{N}$ derived from SCE derived AMPK phosphorylation in steatotic HepG2 cells, that ultimately =>LXR restraint, deactivation of ACC via phosphorylation and the prevention of SREBP1c translocation. In this pathway that has been presented, active LXR, following binding to the retinoid X LXR response elements in their promoters. Hence reduction of triglycerides accumulation in adipocytes and hepatocytes might result from the multifaceted biological activity of SCE.

\section{Aging related actions of SCE}

Besides date of birth cellular senescence can be important part of aging and age related diseases [35]. Aging research includes senescence, direct aging, photoaging, oxidative, mitochondrial, inflammatory aging among others. For studying aging in experimental practice, either cellular replicative senescence is investigated or various animal models of aging are used. D-galactose -induced aging is a commonly applied model of aging [36].

Schisandrin B along with its analogue Schisandrin C, were c shown to protect human and rat foreskin fibroblasts against oxidative damage induced by solar light [37]. These substances were speculated to be used in the prevention of photoaging. Protective effect was conferred by decreased production of reduced glutathione, decreased expression of matrix matelloproteinase 1 and an elastase -type protease. However these compounds also produced ROS during their metabolism, mediated by the cytochrome P-450, and probably this reaction provoked potentiated antioxidant response by the glutathione system. Similar results were obtained for Schisandrin B in the human keratinocyte-derived cell line Ha Ca T. Schisandrin B decreased cell death, DNA damage, and oxida- 
tion of proteins in these cells challenged by oxidative stress, and increased the expression of key enzymes of the antioxidant defense and stimulated the Nrf2 (nuclear factor erythroid 2 related factor 2) and MAPKs(mitogen activated protein kinases)pathways. Same responses were seen for deoxy Schisandrin and Schisandrin $\mathrm{B}$ in Ha Ca T keratinocytes exposed to UVB. In total these effects were considered important for skin aging prevention underlined by oxidative stress.

In the middle aged or elderly, osteoarthritis (OA), a joint disease occurs. An ethanol extract of SCE exerted a positive effect against cartilage degradation in a monosodium iodoacetate (MIA)induced OA rat model [38]. This Protective effect was underlined by a decreased production of inflammatory cytokines and TNF- $\alpha$, an inhibited expression of inducible nitric oxide synthase, and cyclooxygenase 2 and increased levels of MMP-13, cartilage oligomeric matrix protein and $\mathrm{C}$-telopeptide of type II collagen.

Sarcopenia, a progressive loss of muscle strength and mass with aging, is commonly thought of as an important indicator of aging and occurs in some diseases associated with accelerated aging. SCE increased mass of skeletal muscle in mice and rats treated by dexamethasone or those who underwent sciatic neurectomy. Kim., et al. showed that SCE ameliorated muscle atrophy by increased protein synthesis which resulted from downregulation of MTOR/p-4EBP1 (4E-binding protein 1)/p-P70S6K (70kDa ribosomal protein S6kinase) pathway in human myoblasts [38,39]. But SCE can also cause protein degradation via F0X01/MuRF1 pathway but its net action resulted in muscle hypertrophy. Previous report by Kang gave further information on this mechanism by SCE in C2C12 myoblasts [40]. Since aging compromises muscle mass, mitigation of these effects by SCE can be considered as a manifestation of its anti-aging potential. Recently Kim., et al. demonstrated that SCE upregulated genes whose products are important in protein synthesis and muscle growth in old mice after chronic forced exercise swimming [41]. Further SCE downregulated genes important for protein degradation. SCE further decreased the level of ROS and lipid peroxidation, along with upregulating some antioxidant enzymes and inhibited certain apoptotic markers. Hence SCE can be considered as an element to assist exercise based, healthy lifestyle. Further upregulation of PPAR $-\gamma$-coactivator- 1 alpha (PGC- $1 \alpha$ ), and some other proteins in the skeletal muscle of trained animals.
Genisin A, another bioactive compound isolated from SCE, suppressed stress induced premature senescence and the production of pro inflammatory molecules in human fibroblasts [42]. This Protective effect was secondary to the promotion of mitochondrial biogenesis and autophagy by Genisin A in these cells, as well as antioxidant activity. Still some of these works need clarification.

Diet supplementation with Schisandrin B mitigated age-related impairment of mitochondrial antioxidant functions in different tissues of C57BL/6J mice [43]. Thus Schisandrin B increased the survival of aging individuals by improvement of mitochondrial functions. Still despite convincing results on the mutual relationships between aging and antioxidant mitochondrial functions in rats, one cant translate these to humans [44].

Accelerated aging induced in rats by D-galactose, fed with a diet rich in SCE lignans displayed 15 biomarkers having antiaging mechanisms [45]. These markers were involved in energy, amino acids, lipid and phospholipid metabolism, and almost all returned to control levels following termination of SCE lignans supplementation. Further, a SCE lignans rich diet resulted in mRNA overexpression of p19, p53, and p21 proteinsin the brain of aging animals. Hence these metabolic changes in SCE lignans rich diet are secondary to modulation of the expression of these proteins and become a target for antiaging prevention along with therapy.

Further aging is associated with behavioral/cognitive performance besides changes in biochemical functions. D-galactose -induced rats following diet supplementation with ethanol extracts of SCE partitioned with petroleum ether had attenuation of cognitive deficits assayed by the Morris water maze and step -down type passive avoidance test as compared to animals with non-supplemented diet as shown by Yan., et al. [46]. These behavioral changes were associated with a reduced activity of antioxidant enzymes induced by D-galactose and a normal level of oxidative stress markers, including glutathione, malondialdehyde, and nitric oxide in the serum and various structures of the brain of treated animals [46]. Thus SCE extracts and derivatives show benefits against pathological aging as shown in animal models (figure 3). Use in human aging and age related diseases needs to be determined, but they justify further research into antiaging effects of SCE. 


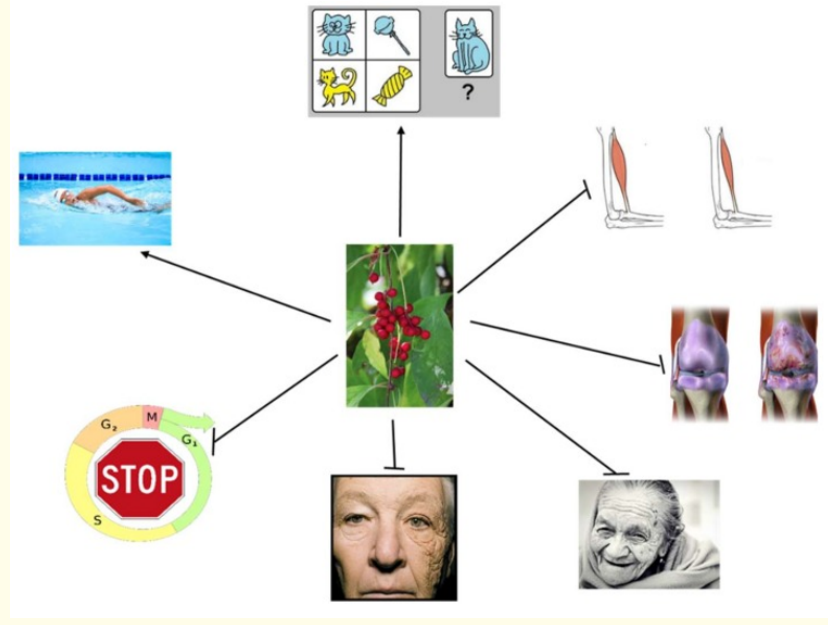

Figure 3

Courtesy ref no.7 Schisandra chinensis and its derivatives can modulate aging-related phenomena in humans, experimental animals and cell cultures. They can suppress skin photoaging, ameliorate sarcopenia and osteoarthritis, increase physical endurance, inhibit stress-induced premature senescence, improve cognitive and behavioural functions, and modulate other effects that can also be associated with a delay of normal aging.

\section{Conclusions}

In our earlier reviews we have reviewed how obesity is becoming a global epidemic with both obesity and T2DM increasing so much that now it is considered to treat them together with the term coined diabesity [47]. The WHO shows that roughly $8 \%$ of world adult population are afflicted with T2DM, and approx $13 \%$ are obese $[48,49]$. Further cancer is the second leading cause of death globally, causing approximately. 6 million deaths in 2018. Main reasons are low fruit and vegetable intake, bacterial and viral infections, and obesity/overweight. Furthermore, in many cases cancer can be considered as an aging disease, though mechanisms correlating the two are not clear.

In view of that we have earlier reviewed role of natural products like flavonoids, role of soya beans and chick peas, walnuts, monoterpenes, PIP1Binhibitors from plants as natural sources for treating obesity $[47,50]$ and on PTPB1 inhibitors under publication]. Here we chose Schisandra chinensis for this review as it is one plant having different biochemical compounds like terpe- noids, flavonoids, PTB1B inhibitors all in one plant, hence seems to be very promising to be further exploited for use in human DM, obesity, cancer prevention along with treatment and prevention of aging, prevention against antimicrobial infections, prevention of DM with its action on GLUT and SGLT2 transporters.

\section{Bibliography}

1. Saxena M., et al. "Phytochemistry of medicinal plants". Journal of Pharmacognosy and Phytochemistry 1 (2013): 168-182.

2. Panossian A and Wikman G. "Pharmacology of Schisendra chinensis Bail: An overview of Russian research and uses in medicine". Journal of Ethnopharmacology 118 (2008): 183-212.

3. Szopa A., et al. "Current knowledge of Schisendra chinensis (Turcz) Bail. (Chingse magnolia vine) as a medicinal plant species: A review on bioactive substances, pharmacological properties, anakytical and biotechnological studies". Phytochemistry Review 16 (2017): 195-218.

4. Li Z., et al. "A review of polysaccharides from Schisendra chinensis and Schisendrasphenathra: Properties, functions and applications". Carbohydrate Polymer 184 (2018): 173-190.

5. Szopa A and Ekiert H. "The importance of applied light quality on the production of lignans and phenolic acids in Schisend ra chinensis (Turcz) Bail cultures in vitro". Plant Cell, Tissue and Organ Culture 127 (2016): 115-121.

6. Yang BY., et al. "New lignans from the rattan stems of Schisendra chinensis". Nature Production Research (2018): 1-7.

7. Nowak A., et al. "Potential of Schisendra chinensis (Turcz) Bail. in Human Health and Nutrition: A Review of current Knowledge and Therapeutic Perspectives". Nutrients 11 (2019): 333.

8. Xia YG., et al. "Schisandracea triterpenoids: A review". Phytochemistry Research 14 (2015): 155-187.

9. Yang S., et al. "Rapid classification and identification of chemical components of Schisendra chinensis by UPLC-Q-TOF/MS combined with data processing". Molecules 72 (2017): 1778.

10. Szopa A., et al. "Studies on the accumulation of phenolic acids and flavonoids in different in vitro culture systems of Schisendra chinensis (Turcz) Bail using a DAD-HPLC method". Phytochemistry Letter 20 (2017): 462-469.

11. Ma C., et al. "Content and color stability of anthocyanins isolated from Schisendra chinensis fruit". International Journal of Molecular Sciences 13 (2012): 14294-14310. 
12. Xu CL., et al. "Inhibitory effects of Schisendra chinensis leaf polysaccharides against L5178Y lymphoma". Carbohydrate Polymer 88 (2012): 21-25.

13. Tong H., et al. "Isolation and phytochemical characterization of polysaccharides fractions isolated from Schisendra chinensis". Chemistry of Natural Compounds 47 (2012): 969-70.

14. Sowndharajan K., et al. "Evaluation of proximate composition, bioactive lignans and volatile composition of Schisendra chinensis fruits from Inje and Mung yeong, Republic of Korea". Journal of Applied Pharmaceutical Science 6 (2016): 001-008.

15. Teng $\mathrm{H}$ and Lee WY. "Antibacterial and antioxidant activities and chemical composition of volatile oil extracted from Schisendra chinensis Baill. seeds using simultaneous distillation extraction method, and comparison with soxhet and microwave assisted extraction". Bioscience, Biotechnology, and Biochemistry 28 (2014): 79-83.

16. Choi EJ., et al. "Antimicrobial activity of Psoralea corylifolia, Schisendra chinensis and Spatiobolus suberectus extracts". Korean Journal of Food Science and Technology 45 (2013): 495-500.

17. Yu H. "Antimicrobial activity and mechanism of Schisendra chinensis extract". In Proceedings of the 5th International Conference on Environment. Materials, Chemistry and Power Electronics (EMCPE 2016). Zhengzhou China. 192-196.

18. Min KJ and Kwon TK. "Anticancer effects and molecular mechanisms of epigallcatechin-3-gallate". Integrative Medicine Research 3 (2014): 16-24.

19. Venkanna A., et al. "Phytochemical investigation of sesquiterpenes from the fruits of Schisendra chinensis and their cytotoxic activity". Fitoterapia 95 (2014): 102-108.

20. Lv XJ., et al. "Schisandrin B inhibits the proliferation of human lung adenocarcinoma A549 cells by inducing cycle arrest and apoptosis". International Journal of Clinical and Experimental Medicine 8 (2015): 6926-6936.

21. Kee JY., et al. "A suppresses colorectal lung metastasis by inducing AMPK/P38-mediated apoptosis and decreasing metastatic abilities of colorectal cells". Fruit Pharmacology 20189 (2018): 986.
22. Casarin E., et al. "Molecular mechanisms of antiproliferative effects induced by Schisendra derived Dibenzocyclooctadiene lignans (+) deoxy scisandrin and (-) -gomisin $\mathrm{N}$ in human tumor cell lines". Fitoterapia 98 (2014): 241-7.

23. Liu Z., et al. "Schisandrin B attenuates cancer invasion and metastasis via inhibiting epithelial -mesenchymal transition". PLOS ONE 7 (2012): e10480.

24. Liu H., et al. "Extracts and lignans of Schisendra chinensis fruit alter lipid and glucose metabolism in vivo and in vitro". Journal of Function Foods 201519 (2015): 296-307.

25. Jo SH., et al. "In vitro and in vivo antihyperglycemic effects of omija (Schisendra chinensis) fruit". International Journal of Molecular Sciences 12 (2011): 1359-1370.

26. Qu Y., et al. "Antidiabetic effect of Schisendra chinensis Fructus involves inhibition of the sodium glucose cotransporter". Drug Development Research 76 (2015): 1-8.

27. Jin D., et al. "Schisendra polysaccharides increased glucose consumption by upregulating the expression of GLUT4". International Journal of Biological Macromolecules 87 (2016): 555-562.

28. Mueckler M and Thorens B. "The SLC2 (GLUT) family of membrane transporters". Molecular Aspects of Medicine 34 (2013): 121-138.

29. Hardie DJG. "Regulation of AMP-Activated protein kinase by natural and synthetic activators". Acta Pharmaceutica Sinica B 6 (2016): 1-9.

30. Fang LL., et al. "Protein tyrosine phosphatase 1B (PTP1B) and $\alpha$-glucosidase-inhibitory activities of Schisendra chinensis (Turcz) Baill”. Journal of Function Foods 9 (2014): 264-270.

31. Zaklos-Szyda M., et al. "Antidiabetic effect of polyphenolic extracts from selected edible plants as $\alpha$-amylase and $\alpha$-glucosidase and PTP1B inhibitors and $\beta$-pancreatic cellscytooprotective agents-A comparative study". Current Topics in Medicinal Chemistry 15 (2015): 2431-2444.

32. Park S., et al. "Huang-Lian-Jie -Du Tang supplemented with Schisendra chinensis Baill and Polygonatum odoratum druce improved glucose tolerance by potentiating insulinotropic actions in islets in 90\% pancreatetomized diabetic rats". Bioscience, Biotechnology, and Biochemistry 73 (2009): 2384-2392. 
33. Kwan HY., et al. "Schisandrin B regulates lipid metabolism in subcutaneous adipocytes". Science Report 17 (2017): 10266.

34. Liu X., et al. "Deoxyschizandrin loaded liposomes on the suppression of lipid accumulation in 3T3L1 adipocytes". Molecules 23 (2018): 2158.

35. Zhu PL., et al. "Effects of combined dietary supplementation with fenofibrate and Schisandrae Fructus pulp on lipid and glucose levels and liver function in normal and hypercholesteraemic mice". Drug Design, Development and Therapy (2015): 923-935.

36. Chung MY., et al. "Schisendra chinensis berry extract protects against steatosis by inducing histone acetylation in oleic acid -treated HepG2 cells and in the livers of diet induced obese mice". Nutrition Research 46 (2017): 1-10.

37. Jeyapalan JC and Sedivy JM. "Cellular senescence and organismal aging". Mechanisms of Ageing and Development 129 (2008): 167-174.

38. Kim JW., et al. "The administration of Fructus Schisandrae attenuates dexamethasone -induced muscle atrophy in mice". International Journal of Molecular Medicine 36 (2015): 29-42.

39. Kim CH., et al. "Schisandrae Fructus enhances myogenic differentiation and inhibits atrophy through protein synthesis in human myotubes". International Journal of Nanomedicine 11 (2016): 2407-2415.

40. Kang JS., et al. "Nrf2 mediated HO-1 induction contributed to antioxidant capacity of Schisandrae Fructus ethanol extract in C2Cl2 myoblasts". Nutrients 6 (2014): 5667-5578.

41. Kim JW., et al. "Muscle protective effects of Schisandrae Fructus extracts in old mice after chronic forced exercise". Journal of Ethnopharmacology 212 (2018): 175-187.

42. Kim JS., et al. "Gomisin A modulates aging process via mitochondrial biogenesis in human diploid fibroblast cells". Clinical and Experimental Pharmacology and Physiology 45 (2018): 547-555.

43. Ko KM., et al. "Long term Schisandrin B treatment mitigates age related impairments in mitochondrial antioxidant status and functional ability in various tissues and improves the survival of aging C57BL/6J mice". Biofactors 34 (2008): 331-342
44. Meng Q., et al. "Age related changes in mitochondrial function and antioxidant enzyme activity in Fischer 344 rats". Mechanisms of Ageing and Development 128 (2007): 286-292.

45. Sun I., et al. "Metabolomic study of the therapeutic mechanism of Schisendra chinensis lignans on aging rats induced by Dgalactose". Clinical Interventions in Aging 13 (2018): 829-841.

46. Yan T., et al. "Lignans from Schisendra chinensis ameliorate cognitive deficits and attenuates brain oxidative damage induced by D-galactose in rats". Metabolic Brain Disease 31 (2016): 653-661.

47. Kulvinder Kochar Kaur., et al. "Importance of simultaneous treatment of obesity and diabetes mellitus: A sequelae to the understanding of diabesity-A review". Obesity Research 6 (2019): 1-10.

48. World Health Organization. "Global Report on Diabetes" (2016).

49. World Health Organization. "Obesity and Overweight-Key Facts" (2018).

50. Kulvinder Kochar Kaur., et al. "Monoterpenes -A Class of Terpenoid Group of Natural Products as an Source of Natural Antidiabetic Agents in the Future -A Review". CPQ Nutrition 3 (4): 01-21.

\section{Volume 3 Issue 8 August 2019 (C) All rights are reserved by Kulvinder Kochar Kaur., et al.}

